\title{
CPR and YOU
}

\section{What every medical student needs to know about performing CPR}

\author{
Lauren Prufer (Meds 2016) \\ Faculty Reviewer: Dr Dan Howes, MD, FRCPC (Departments of Emergency Medicine and Critical Care)
}

\section{BACKGROUND}

Due to the difficulties associated with recording and analyzing low-frequency emergency events, in-hospital cardiac arrest (IHCA) intervention has historically been less rigorously studied than out-of-hospital cardiac arrest (OHCA). Survival of IHCA is low: reported survival to hospital discharge (SHD) rates range from $6.6 \%$ to $18 \%$, with $5.2 \%$ of patients surviving to one year. ${ }^{1-4}$ Survival rates have not significantly improved in decades, ${ }^{5}$ and studies of in-hospital cardiopulmonary resuscitation (CPR) quality have found that CPR provided by trained in-hospital staff often does not conform to published American Heart Association (AHA) guidelines. ${ }^{6}$ Hesitancy of first responders, typically nurses and residents, to initiate CPR in the absence of an attending physician, ${ }^{7}$ despite measurable survival benefits associated with rapid initiation of CPR and other resuscitation system errors, ${ }^{8-13}$ such as delayed or incorrect medication administration,14 may also contribute to reduced survival rates for IHCA. Studies of skill acquisition and retention in medical students have demonstrated that CPR proficiency deteriorates rapidly over time, resulting in suboptimal CPR quality upon entry into a clinical setting. ${ }^{15}$ This paper aims to familiarize medical learners with basic indicators of CPR quality.

\section{INDICATORS OF CPR QUALITY FOR MEDICAL LEARNERS}

Indicators of CPR quality have been linked to increased likelihood of return of spontaneous circulation (ROSC) and improved SHD and include time to respond, chest compression fraction (CCF), rate of compression, chest compression depth and leaning and ventilation.

\section{Time to Respond}

Time to respond is the time from cardiovascular collapse to the initiation of chest compressions or defibrillation. Rapid initiation of CPR is important for the maintenance of organ and tissue perfusion during cardiac arrest (CA). ${ }^{9}$ Two groups have found that odds of SHD were approximately halved when CPR and defibrillation was initiated more than 60 seconds postcollapse, ${ }^{11,12}$ and several groups have observed significant correlations between decreased survival rate and increased response time. ${ }^{13,16}$

Current AHA recommendations state that teams should initiate compressions and defibrillation less than 120 seconds following collapse. ${ }^{8}$ Studies on IHCA response times found an average of 180 seconds elapsed before response team arrival. ${ }^{7}$ As well, once the team had arrived, a further 35 seconds elapsed before initiation of CPR. ${ }^{17}$ First responders, typically residents or nurses, were hesitant to check pulse, analyze rhythm and initiate CPR or defibrillation in the absence of an attending physician. ${ }^{7}$ Trained and qualified first responders failed to initiate CPR and defibrillation $12 \%$ and $44 \%$ of the time, respectively. ${ }^{7}$ The rate of SHD following IHCA at this institution did not differ significantly from national averages, suggesting that reluctance of trained ward staff to initiate treatment prior to arrival of the emergency response team could be contributing to the low survival rates observed across institutions. ${ }^{7}$

\section{Chest Compression Fraction (CCF)}

CCF is the proportion of total time from CA to ROSC or death spent performing chest compressions. Clinical studies have shown that a higher CCF increases the likelihood of ROSC and SHD. ${ }^{19-22}$ A prospective observational study of ventricular tachycardia (VT) and ventricular fibrillation (VF) found that every $10 \%$ increase in CCF resulted in a 1.11-fold increased odds ratio (OR) of SHD. ${ }^{19}$ Minimal-pause CPR protocols have also been widely studied; reduced pre-, peri- and post-shock pause and increased CCF have been associated with improved SHD. ${ }^{20-22}$ A trial using mechanical chest compressions found decreased likelihood of cerebrovascular incidents and increased likelihood of ROSC and SHD with use of a minimal-pause CPR protocol. ${ }^{23}$ AHA guidelines suggest that CA response teams use a minimal-pause protocol and aim for a CCF of greater than $0.8^{8}$

\section{Rate of Compression}

2010 AHA guidelines recommend a rate of greater than 100 compressions per minute. ${ }^{24}$ Data from the Resuscitation Outcomes Consortium (ROC) Cardiac Arrest Epistry suggest that compression rates follow a dose-dependent curve, finding that rates between 100 and 125 compressions per minute have been associated with the highest likelihood of ROSC. ${ }^{25}$ Rates lower than 100 may reduce tissue oxygenation and rates greater than 120 may compromise compression depth and coronary artery perfusion (CAP). ${ }^{27,28}$ Compressions are often performed too slowly, with one study finding average rates of less than 80 in $36.9 \%$ of resuscitation episodes. ${ }^{29}$ Receiving less than 80 compressions per minute was associated with a $30 \%$ reduction in likelihood of attaining ROSC. ${ }^{29}$

\section{Chest Compression Depth and Residual Leaning}

Chest compression depth measures the sternal displacement during compressions while residual leaning refers to continued pressure on the chest between compressions. The AHA recommends a compression depth of at least $50 \mathrm{~mm}$ in adults and minimal residual leaning. ${ }^{9}$ 
Compression depth greater than $50 \mathrm{~mm}$ during the 30 seconds before defibrillation has been associated with increased likelihood of successful defibrillation, ROSC and SHD. ${ }^{10,25}$ Despite the importance of compression depth in maintaining adequate perfusion, studies of out-of-hospital CPR have found that suboptimal compressions during CPR are common. One observational study of Canadian and American hospitals found that compressions performed by trained emergency medical responders did not meet the 2010 AHA guidelines $91 \%$ of the time. ${ }^{10}$

Residual leaning between compressions may result in reduced cardiac output due to inhibition of venous return. ${ }^{30-32}$ Studies in porcine models have demonstrated that residual pressure between compressions may be associated with increased right atrial pressure, decreased coronary and cerebral artery perfusion and poor neurological outcome. ${ }^{30-32}$ Studies of human rescuers have found that leaning was present during half of recorded compressions. ${ }^{33}$ Rescuers may be more prone to leaning if they are very tall or perform compressions using a stool. ${ }^{34}$

\section{Ventilation}

Rate and magnitude of ventilation are important indicators of CPR quality. ${ }^{19,35,36}$ AHA guidelines recommend a ventilation rate of less than 12 breaths per minute and that rescuers refrain from overinflating lungs. ${ }^{10}$ Physiologic studies using the porcine model found that overventilation resulted in decreased CAP and venous return to the heart as well as decreased survival. ${ }^{19,35}$ End tidal $\mathrm{CO}_{2}$ $\left(\mathrm{ETCO}_{2}\right)$ concentrations may also be used as an indicator of CPR quality and tissue perfusion during IHCA. ${ }^{37,38}$ The AHA expert panel has recommended that $\mathrm{ETCO}_{2}$ not fall below $10 \mathrm{mmHg}$ during $\mathrm{CPR}$ and that abrupt increase in $\mathrm{ETCO}_{2}$ to greater than 35 to 40 $\mathrm{mmHg}$ be considered an indicator of ROSC. ${ }^{8}$

\section{KEY POINTS FOR MEDICAL LEARNERS DURING ICHA}

Resuscitation errors in the hospital setting are common, with one study of 118,387 IHCAs reporting that $40.4 \%$ of in-hospital VF/ pulseless VTs were associated with a resuscitation system error. ${ }^{38}$ Delayed defibrillation and chest compression were among the most common errors recorded in the in-hospital setting. ${ }^{38}$ Based on hazard ratio analysis, the presence of a documented error was associated with a $34.2 \%$ (95\% confidence interval, $29.5-39.1)$ increase in relative risk of death prior to hospital discharge. ${ }^{38}$ Medical students can contribute to improved patient outcome by educating themselves about signs and symptoms of impending CA (Figure), ${ }^{39,40}$ activation of in-hospital emergency call systems, location of AED devices and necessary equipment on each hospital floor and through prompt initiation of high-quality CPR. CPR providers should "push fast, push hard", aiming for rates greater then 100 compressions per minute and compression depth greater then $50 \mathrm{~mm} .{ }^{8,24} \mathrm{Com}-$ pression quality may be improved by practicing with a metronome or automated feedback device; these have been shown to improve both pace and depth of chest compressions in medical learners. ${ }^{41-44}$ Compression depth can be improved through placement of a backboard or use of a hard surface during CPR. ${ }^{8}$ Performing chest compressions using a stool or while kneeling has also been shown to reduce rescuer fatigue by increasing mechanical advantage; ${ }^{34,45}$ however, care should be taken to avoid leaning. Frequent rotation of rescuers at prespecified intervals may also help to reduce fatigue and improve compression quality.

Pauses in CPR can be minimized by setting specific time goals for task completion (Table). Witnesses to the arrest should communicate all pertinent information succinctly upon team leader arrival. Students who have access to a dummy and wish to practice with feedback may be interested in downloading an automated feedback device to their smartphone, such as the ZOLL pocket CPR device. Though not recommended for use during real-life situations, the program is capable of giving automated feedback about compression rate and depth and may give students valuable practice by helping to improve CPR quality during simulation.

\begin{tabular}{|c|c|c|c|c|c|c|c|}
\hline \multicolumn{8}{|c|}{ National Early Warning Score (NEWS) } \\
\hline $\begin{array}{l}\text { PHYSIOLOGICAL } \\
\text { PARAMETERS }\end{array}$ & 3 & 2 & 1 & 0 & 1 & 2 & 3 \\
\hline Respiration Rate & $\leq 8$ & & $9-11$ & $12-20$ & & $21-24$ & $\geq 25$ \\
\hline $\begin{array}{c}\text { Oxygen } \\
\text { Saturations }\end{array}$ & $\leq 91$ & $92-93$ & $94-95$ & $\geq 96$ & & & \\
\hline $\begin{array}{l}\text { Any Supplemental } \\
\text { Oxygen }\end{array}$ & & Yes & & No & & & \\
\hline Temperature & $\leq 35.0$ & & $35.1-36.0$ & $36.1-38.0$ & $38.1-39.0$ & $\geq 39.1$ & \\
\hline Systolic BP & $\leq 90$ & $91-100$ & $101-110$ & $111-219$ & & & $\geq 220$ \\
\hline Heart Rate & $\leq 40$ & & $41-50$ & $51-90$ & $91-110$ & $111-130$ & $\geq 131$ \\
\hline $\begin{array}{l}\text { Level of } \\
\text { Consciousness }\end{array}$ & & & & A & & & $\mathrm{V}, \mathrm{P}$, or U \\
\hline
\end{tabular}

The National Early Warning Score (NEWS) thresholds and triggers

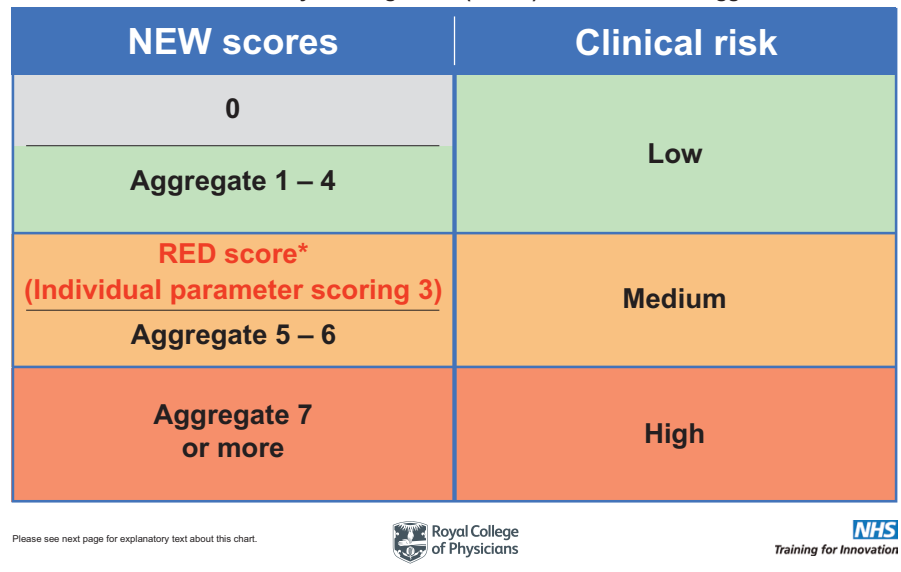

Figure: Sample of scoring system for physiological deterioration in-hospital. Increasing national early warning score (NEWS) is indicative of increasing risk of death, ICU admission or CA within next $24 \mathrm{hrs} .40 \mathrm{~A}$ level of conciousness score of $A$ is assigned if the patient is alert. A score of $V, P$ or $U$ is assigned if a patient responds only to voice or pain or is unresponsive. Patients may be stratified into clinical risk categories using NEWS. Patients with an aggregate NEWS of greater then 7 or any individual parameter scoring greater then 3 are considered to be at high risk and require urgent clinical evaluation. Clinical tools like the NEWS system can help to identify high risk patients, allowing for early intervention and thus prevention of adverse events. The NEWS can be found at http://www.rcplondon.ac.uk/resources/national-early-warning-score-news. 
Table: Summary of Components of High-Quality CPR for Medical Learners

\section{QUALITY INDICATOR}

\begin{tabular}{|c|c|}
\hline 1. General & $\begin{array}{l}\text { - Be aware of and monitor patients for signs and symptoms of physiological deterioration which may } \\
\text { - Krecede cardiac arrest (see Table 1). } \\
\text { - Know how to activate emergency response if an unconscious patient is discovered. } \\
\text { - Knon of AEDs and other required medical equipment. }\end{array}$ \\
\hline 2. Time to respond & - Rapid initiation of chest compressions. \\
\hline 3. Chest Compression Fraction & $\begin{array}{l}\text { - Keep pauses during CPR to a minimum: } \\
\text { - Less than } 10 \text { s for rhythm analysis and defibrillation. } \\
\text { - Less than } 3 \text { s to rotate compressors. } \\
\text { - Rotate compressors regularly during necessary pauses for ventilation. }\end{array}$ \\
\hline 4. Chest Compression Rate & - 100-120 per minute. \\
\hline 5. Chest Compression Depth & $\begin{array}{l}\text { - }>50 \text { mm. } \\
\text { - Use a backboard or hard surface. } \\
\text { - Use a stool or kneel while performing compressions. } \\
\text { - Avoid leaning between compressions. } \\
\text { - Rotate compressors frequently to avoid fatigue. }\end{array}$ \\
\hline 6. Ventilation & $\begin{array}{l}\text { - Rate of }<12 \text { breaths per minute. } \\
\text { - Avoid overinflating lungs. }\end{array}$ \\
\hline
\end{tabular}

\section{REFERENCES}

1. Hodgetts TJ, et al. Incidence, location and reasons for avoidable in-hospital cardiac arrest in a district general hospital. Resuscitation. 2005(2);120:158-64.

2. Jones-Crawford JL, Parish DC, Smith BE, Dane FC. Resuscitation in the hospital: circadian variation of cardiopulmonary arrest. Am J Med. 2010;170(2):18-26.

3. Nadkarni VM, et al; National Registry of Cardiopulmonary Resuscitation Investigators. First documented rhythm and clinical outcome from in-hospital cardiac arrest among children and adults. JAMA. 2006;295(1):50-7.

4. Bloom HL, et al. Long-term survival after successful in-hospital cardiac arrest resuscitation. AM Heart J. 2007;153(5):831-6.

5. Peberdy MA, et al. Cardiopulmonary resuscitation of adults in the hospital: a report of 14270 cardiac arrests from the National registry of cardiopulmonary resuscitation. Resuscitation. 2003;58(3):297-308.

6. Abella BS, et al. Chest compression rates during cardiopulmonary resuscitation are suboptimal a prospective study during in-hospital cardiac arrest. Circulation. 2005;111(4):428-34.

7. Einav S, et al. Performance of department staff in the window between discovery of collapse to cardiac arrest team arrival. Resuscitation. 2006;69(2):213-20.

8. Meaney PA, et al. CPR Quality: Improving cardiac resuscitation outcomes both inside and outside the hospital a consensus statement from the American Heart Association. Circulation. 2013;128(4):417-35.

9. Stiell IG, et al; Resuscitation Outcomes Consortium (ROC) Investigators. What is the role of chest compression depth during out-of-hospital cardiac arrest resuscitation? Crit Care Med. 2012;40(4):1192-8.

10. De Maio VJ, et al. Optimal defibrillation response intervals for maximum out-of-hospital cardiac arrest survival rates. Annals of emergency medicine. 2003;42(2): 242-50.

11. Chan PS, et al. Delayed time to defibrillation after in-hospital cardiac arrest. New Engl J Med. 2008;358(1): 9-17.

12. Herlitz J, et al. Characteristics and outcome among patients suffering from in hospital cardiac arrest in relation to the interval between collapse and start of CPR. Resuscitation. 2002;53(1): 21-7.

13. Herlitz J, et al. Very high survival among patients defibrillated at an early stage after in-hospital ventricular fibrillation on wards with and without monitoring facilities. Resuscitation. 2005;66(2):159-66.

14. Ornato JP, et al. Impact of resuscitation system errors on survival from in-hospital cardiac arrest. Resuscitation. 2012;83(1):63-9.

15. Behrend T, et al. Retention of cardiopulmonary resuscitation skills in medical students utilizing a high-fidelity patient simulator. Medical Student Research Journal. 2011;1(1):1-4.

16. De Maio VJ, et al. Optimal defibrillation response intervals for maximum out-of-hospital cardiac arrest survival rates. Ann Emerg. 2003;42(2):242-50.

17. Marsch SCU, et al. Performance of first responders in simulated cardiac arrests. Crit Care Med. 2005;33(5):963-7.

18. Berg RA, Sanders AB, Kern KB, et al. Adverse hemodynamic effects of interrupting chest compressions for rescue breathing during cardiopulmonary resuscitation for ventricular fibrillation cardiac arrest. Circulation. 2001;104(20):2465-70.

19. Christenson J, et al. Resuscitation Outcomes Consortium Investigators. Chest compression fraction determines survival in patients with out-of-hospital ventricular fibrillation. Circulation. 2009;120(13):1241-7.

20. Cheskes $\mathrm{S}$, et al. The impact of peri-shock pause on survival from out-of-hospital shockable cardiac arrest during the resuscitation outcomes consortium PRIMED trial. Resuscitation 


\section{FEATURE ARTICLE}

[Internet]. 2013 Oct 28 [cited Nov 15]. Available from: http:// www.resuscitationjournal.com/article/S0300-9572(13)008149/abstract.

21. Rea TD, Helbock M, Perry S, et al. Increasing use of cardiopulmonary resuscitation during out-of-hospital ventricular fibrillation arrest: survival implications of guideline changes. Circulation. 2006;114(25):2760-5.

22. Eftestøl T, Kjetil S, Steen PA. Effects of interrupting precordial compressions on the calculated probability of defibrillation success during out-of-hospital cardiac arrest. Circulation. 2002;105(19): 2270-3.

23. Lee I, et al. Improved survival outcome with continuous chest compressions with ventilation compared to $5: 1$ compressions-to-ventilations mechanical cardiopulmonary resuscitation in out-of-hospital cardiac arrest. J Chin Med Assoc. 2013;76(3):158-63.

24. Edelson DP, Abella BS, Kramer-Johansen J, et al. Effects of compression depth and pre-shock pauses predict defibrillation failure during cardiac arrest. Resuscitation 2006;71(2):137-45.

25. Idris $\mathrm{AH}$, et al. Relationship between chest compression rates and outcomes from cardiac arrest clinical perspective. Circulation. 2012;125(24):3004-12.

26. Monsieurs KG, et al. Excessive chest compression rate is associated with insufficient compression depth in prehospital cardiac arrest. Resuscitation. 2012;83(11):1319-23.

27. Wolfe JA, Maier GW, Newton JR et al. Physiologic determinants of coronary blood flow during external cardiac massage. J Thorac Cardiovasc Surg. 1988;95(3):523-32.

28. Abella BS, et al. Chest compression rates during cardiopulmonary resuscitation are suboptimal: a prospective study during in-hospital cardiac arrest. Circulation. 2005;111(4):428-34.

29. Zuercher M, et al. Leaning during chest compressions impairs cardiac output and left ventricular myocardial blood flow in piglet cardiac arrest. Crit Care Med. 2010;38(4):1141.

30. Udassi JP, et al. Novel adhesive glove device (AGD) for active compression-decompression (ACD) CPR results in improved carotid blood flow and coronary perfusion pressure in piglet model of cardiac arrest. Resuscitation. 2012;83(6):750-4.

31. Metzger AJ, et al. Improved cerebral perfusion pressures and 24-hr neurological survival in a porcine model of cardiac arrest with active compression-decompression cardiopulmonary resuscitation and augmentation of negative intrathoracic pressure. Crit Care Med. 2012;40(6): 1851-6.

32. Niles D, et al. Leaning is common during in-hospital pediatric CPR, and decreased with automated corrective feedback. Resuscitation. 2009;80(5):553-7.

33. Edelson DP, et al. The impact of a step stool on cardiopulmonary resuscitation: a cross-over manikin study. Resuscitation. 2012;83(7):874-8.

34. Aufderheide TP, Sigurdsson G, Pirrallo RG, et al. Hyperventilation-induced hypotension during cardiopulmonary resuscitation. Circulation. 2004;109(16):1960-5.

35. Langhelle A, Sunde K, Wik L, Steen PA. Arterial blood-gases with 500- versus 1000-ml tidal volumes during out-of-hospital
CPR. Resuscitation. 2000;45(1):27-33.

36. Villanueva-Reiakvam, S; ZOLL Medical Corporation. The ART of Resuscitation. Code Communications Newsletter [Internet]. 2013 [cited Jul]. Available from: http://www.zoll.com/uploadedFiles/Public_Site/Core_Technologies/Real_CPR_Help/ ArtofResuscitation.pdf

37. Myatra SN, Todi AT, Jigeeshu VD. ICU Protocols. XXVI. India: Springer India; 2012. 879 p.

38. Ornato JP, et al. Impact of resuscitation system errors on survival from in-hospital cardiac arrest. Resuscitation. 2012;83(1);63-9.

39. Bellomo R, Goldsmith D, Uchino S, et al. A prospective before-and-after trial of a medical emergency team. Med $\mathrm{J}$ of Aust. 2003;179(6):283-7.

40. Smith GB, et al. The ability of the National Early Warning Score (NEWS) to discriminate patients at risk of early cardiac arrest, unanticipated intensive care unit admission, and death. Resuscitation. 2013;84(4):465-70.

41. Allan KS, Wong N, Aves T, Dorian P. The benefits of a simplified method for CPR training of medical professionals: A randomized controlled study. Resuscitation. 2010;84(8):1119-24.

42. Chung TN, et al. The specific effect of metronome guidance on the quality of one-person cardiopulmonary resuscitation and rescuer fatigue. J Emerg Med. 2012;43(6):1049-54.

43. Martin P, et al. Real-time feedback can improve infant manikin cardiopulmonary resuscitation by up to $79 \%$-a randomized controlled trial. Resuscitation. 2013 84(8):1125-30.

44. Kim SC, et al. A simple audio-visual prompt device can improve CPR performance. J Emerg Med. 2013;44(1):128-34.

45. Foo NP, Chang JH, Lin HJ, Guo HR. Rescuer fatigue and cardiopulmonary resuscitation positions: a randomized controlled cross-over trial. Resuscitation. 2010;81(5):579-84. 